\title{
The spatial structure of European wind storms as characterized by bivariate extreme-value Copulas
}

\author{
A. Bonazzi, S. Cusack, C. Mitas, and S. Jewson \\ Risk Management Solutions, Peninsular House, 30 Monument Street, London, UK \\ Correspondence to: A. Bonazzi (abonazzi@rms.com) \\ Received: 20 October 2011 - Revised: 4 March 2012 - Accepted: 30 March 2012 - Published: 29 May 2012
}

\begin{abstract}
The winds associated with extra-tropical cyclones are amongst the costliest natural perils in Europe. Re/insurance companies typically have insured exposure at multiple locations and hence the losses they incur from any individual storm crucially depend on that storm's spatial structure. Motivated by this, this study investigates the spatial structure of the most extreme windstorms in Europe. The data consists of a carefully constructed set of 135 of the most damaging storms in the period 1972-2010. Extreme value copulas are applied to this data to investigate the spatial dependencies of gusts.

The copula method is used to investigate three aspects of windstorms. First, spatial maps of expected hazard damage between large cities and their surrounding areas are presented. Second, we demonstrate a practical application of the copula method to benchmark catalogues of artificial storms for use in the re/insurance sector. Third, the copula-based method is used to investigate the sensitivity of spatially aggregated damage to climate variability. The copula method allows changes to be expressed in terms of storm frequency, local intensity, and storm spatial structure and gives a more detailed view of how climate variability may affect multilocation risk in Europe.
\end{abstract}

\section{Introduction}

The winds of the most severe extra-tropical cyclones in Europe can cause both loss of life and significant economic damage. For example, Swiss Re (2010) reports about a hundred people either dead or missing in both storm Daria on 25 January 1990 and storm Lothar on 26 December 1999 and several storms in the past $40 \mathrm{yr}$ have caused insured losses in excess of 10 billion US dollars (based on Munich
Re's NATHAN database trended to 2008 values by Barredo, 2010). Barredo (2010) and Klawa and Ulbrich (2003) show evidence that this storm severity is combined with a frequency to produce large average annual losses. These significant impacts from European windstorms generate much interest in risk management.

Probabilistic risk assessment is generally based on the convolution of hazard, vulnerability, and economic or insured exposure (Petak and Atkisson, 1982). Applications of risk management require information on extreme events to answer questions such as: (1) what construction codes are required to protect against winds of return period (RP) of $50 \mathrm{yr}$ ? and (2) what are the capital requirements in re/insurance for robustness to $200 \mathrm{yr}$ return period events? Empirical estimates of such risks are rather inaccurate due to the brevity of the record of reliable observations ( 30 or $40 \mathrm{yr}$ at most), and alternative methods are needed to answer the above questions.

Catastrophe models - generally trade secret vendor products - provide their users with a stochastic set of events that expands the scope of the historical catalogue by including synthetic events that are likely to happen in a defined timeframe. Good agreement between the stochastic catalogue and the historical record distribution of the peril - and perhaps its climate trends - are a necessary condition for confidence in the model's ability to extrapolate to longer return periods.

Independent tests of the hazard component of a catastrophe model are usually conducted before damage assessment. Such independent tests of model components increase confidence in the fidelity of the whole model. The features of the stochastic catalogue of storms that need to be assessed depends on how the model is used. Some sectors, such as construction, are concerned with hazard extremes at specific locations. Friederichs et al. (2009) is an example of 
model validation based on the univariate distribution of wind speeds. However, in the re/insurance sector, both a company's exposure and actual damages occur at multiple locations and hence the spatial structure of individual storms is also relevant.

Insurance decisions are very sensitive to assumptions about the spatial dependence structure of claims data, and a faulty assumption of independence could lead to mis-pricing of policies (e.g. Wang, 1998; Cebrian et al., 2003). Loss estimation tools need to consider various sources of spatial dependence. For instance, models of vulnerabilities of buildings might exhibit a form of dependence structure arising from common building practices in a certain geographical area, while wind-speeds exhibit dependencies because of the spatial structures of storms.

Della-Marta et al. (2008) employed a scalar index of the integral of storm intensity over the whole spatial domain to address this need to capture both the local intensity and the spatial characteristics of a windstorm. However, scalar measures cannot differentiate all the storm characteristics relevant to insurance pricing, in at least two respects. First, extensive damage in recent history has been caused both by extra-tropical storms with a large footprint of relatively low damage ratios (e.g. Kyrill in January 2007 or Jeanette in October 2002), and by storms with smaller areas of intense local damage (e.g. Anatol in December 1999 or 87J in October 1987). Scalar measures of storm intensity cannot capture these different combinations of spatial scale and local intensity. Second, whilst the most intense storms generally travel in the west-to-east direction, there have been notable exceptions, such as 87J and Wiebke in February/March 1990, and a scalar measure of storm intensity cannot resolve these differences in storm path. Condensing information into a scalar measure inevitably fails to capture the full spatial details of storm intensity. The pricing of risk in the re/insurance sector would benefit from a more detailed picture of the spatial structure of European windstorm damage.

The aim of our current work is to explore the spatial characteristics of historical storms in detail, using multivariate estimation of extreme gusts. The results of such an analysis can provide a useful point of comparison with output from stochastic models of storms. Section 2 contains a description of the windstorm data and events used as the basis of our work. The method of multivariate estimation of extreme gusts is presented in Sect. 3. Results from this method are presented in Sect. 4, together with an analysis of sensitivity of joint damaging hazard to climate variability. A comparison of results from this multivariate method with those from an alternative method are presented. Finally, a summary is provided in Sect. 5.

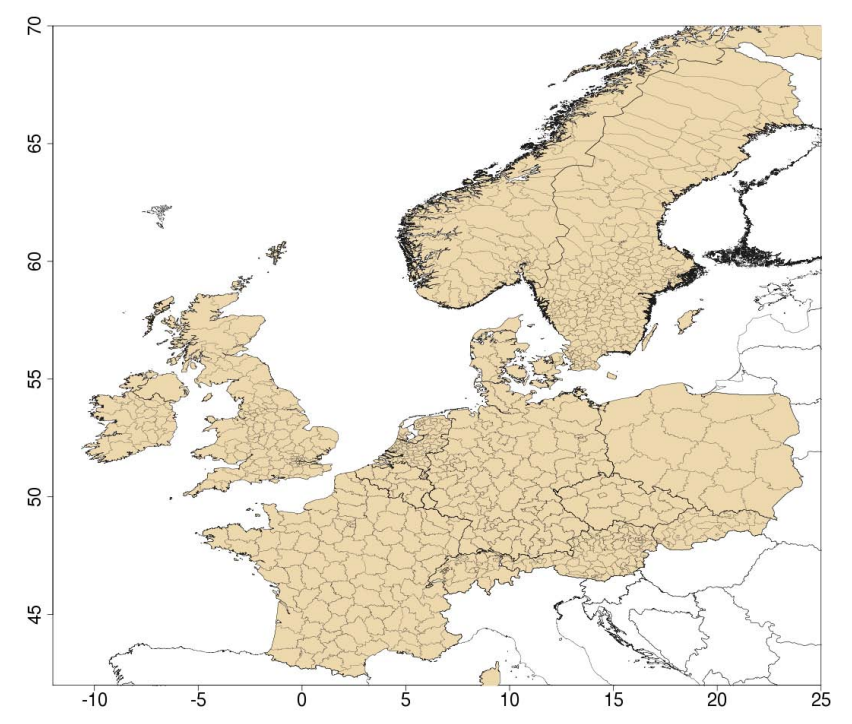

Fig. 1. Analysis domain is formed by 15 European countries. Each countries is further divided in regions known as CRESTA (www. cresta.org) defined with respect to the distribution of population and buildings. CRESTAs generally correspond to 2 digit postal codes.

\section{Description of data}

The overall process of obtaining and processing the basic data for this study can be described as follows. The $3 \mathrm{~s}$ peak gust data were obtained from various sources for the period 1972-2010, covering the 15 European countries shown in Fig. 1. These data were the basis for making storm footprints, which are maps of local maximum gusts during a synoptic storm. These storm footprints were created uniquely for a total of 135 storm events in the study period. The study of the spatial structure of European windstorms in later sections was based on this set of 135 historical storms. The peak gust data and the construction of storm footprints are described in greater detail below.

\subsection{Peak gust data}

We now describe the various sources of peak gust data used in this study. First, peak gust and 10-min wind speed data for ten of the most significant European Wind Storms in the past $25 \mathrm{yr}$ were purchased from MDA Federal Inc. These storms are as follows: 87J, Daria, Vivian, Anatol, Lothar, Martin, Erwin/Gudrun, Kyrill, Emma and Klaus. The data consist of hourly maximum values for a 3 or 5 day window centered on the storm. The observations were obtained directly from national meteorological services and include data from stations not transmitted on the Global Telecommunications System (GTS). There are between 500 and 1000 stations per storm, and MDA applied detailed quality control procedures to the data.

Second, a set of peak gusts was purchased from the UK Met Office (UKMO). This contained 700 stations spread over 
the 15 European countries, and is the largest of the data-sets used. Most station time-series span 1984 to 2009, though some British stations extend back to the start of our study period in 1972. The UKMO data set contains a total of more than four million $3 \mathrm{~s}$ peak gust measurements, and over five million measurements of the daily maximum $10 \mathrm{~min}$ wind speed. The non-UK data are collected from the GTS and are archived digitally with no QC procedures applied. However, QC procedures have been applied to the UK station data.

Third, data was obtained from the Global Summary of the Day (GSOD). GSOD is a freely available data set provided by the National Climate Data Center in the United States and is described in the following webpage: http:// www7.ncdc.noaa.gov/CDO/GSOD-DESC.txt. This data set contains time-series of daily peak gusts and 10-min wind speeds for about 300 stations from 1973 onwards and 600 or more stations from about 1982 onwards. Quality control procedures have been applied to all gust measurements, as described on the web page. Whilst details of the QC procedures are not specified, a comparison with independent and high quality peak gust data from MDA at city locations for some major storms confirmed the reliability of GSOD data.

Fourth, peak gust data were purchased for two of the most recent European windstorms, Klaus (January 2009) and Xynthia (February 2010). The data were purchased from private commercial providers who source the data directly from national meteorological centers. The data for each storm consists of hourly peak gust readings for several hundred stations, including many not transmitted on the GTS.

Fifth, country-specific measurements were obtained from three national meteorological offices. Daily time-series of winds for 44 German stations, most of which begin earlier than 1970 and all of which continue to the present day, are freely available from the Deutscher Wetterdienst (DWD) web site. The Koninklijk Nederlands Meteorogisch Instituut (KNMI) make data for 34 stations located in the Netherlands available for free download from their web site. Most KNMI daily time series begin more than $30 \mathrm{yr}$ ago and all continue to the present day. The daily maximum peak gusts and winds at weather stations in Norway were retrieved from the Norwegian National Meteorological Center (DNMI) web site. In general, DNMI has about 40 stations with daily measurements in the 1980s and increasing to 60 stations in the past 10 or 15 yr. Data for 10 major storms affecting Norway in the past $30 \mathrm{yr}$ were retrieved from the DNMI web site to supplement the data from MDA, GSOD and UKMO in this country.

Finally, smaller data sets were purchased to expand the peak gust data sets where possible. The top 100 gusts in the period 1994 to 2009 at each of 50 stations in Sweden were provided by Sveriges Meteorologiskaoch Hydrologiska Institute (SMHI). MetNext, the commercial branch of Meteo France, provided data for 70 dispersed stations in France from 1980 to 1984, 27 German, 1 Danish and 1 Luxembourg station for 1971 to 1990 , and 3 Irish stations from 1971 to

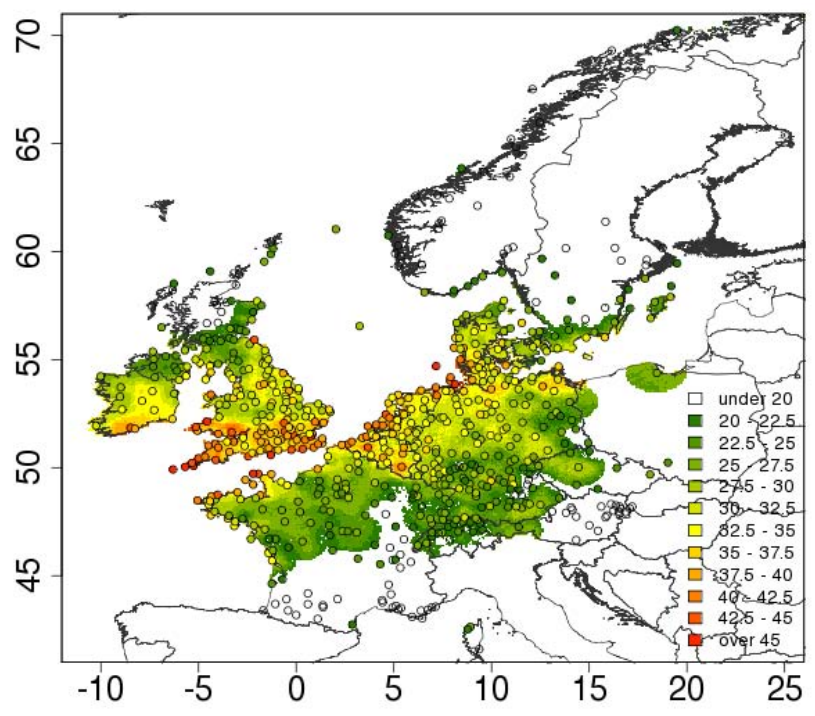

Fig. 2. Map of $3 \mathrm{~s}$ peak gusts $\left[\mathrm{m} \mathrm{s}^{-1}\right]$ at the variable resolution grid (VRG) for storm Daria on 25 January 1990. Circles represent the location of weather stations. Note that gusts at the VRG are weighted towards areas with exposure, hence rougher surfaces than station measurements which tend to be over open terrain, leading to a $10 \%$ reduction in gust strengths in general.

1984. These additional 102 stations improved data coverage where it was considered necessary.

In addition to obtaining direct measurements of peak gusts, some peak gusts were estimated from 10 min windspeeds. This approach was only used when we considered that the uncertainties in local gust estimates due to low data density were higher than the uncertainties due to estimating from $10 \mathrm{~min}$ winds. The conversion from $10 \mathrm{~min}$ winds to peak gusts was based on local site coefficients estimated from satellite-derived land-use and land-cover data. The MDA records of 10-min winds were used, although this data set had almost complete gust records and 87J in north-west France was the only notable exception. The UKMO $10 \mathrm{~min}$ winds in Norway, Sweden and Poland were used to create pseudogusts. GSOD 10-min winds and were used in data-sparse areas of Europe in the 1970s and 1980s, for particular storms (Capella, 24 November 1981, 19 January 1986, 2 November 1981, 14 November 1978, 8 February 1981, 13 January 1984 and 18 January 1983).

The ultimate combined data set consists of around 1500 stations spread across Europe with typical distance between stations of around 50 to $100 \mathrm{~km}$ in the domain, with higher station densities in more urbanized areas such as in Germany, the UK, and France. The circles in Fig. 2 denote the location of station observations during storm Daria. This distribution of weather stations is representative for the regions affected by Daria. The regions outside the damage swath of Daria would tend to have many more reporting stations during a damaging storm than depicted in Fig. 2. 


\subsection{Storm footprint data set}

Our overall strategy is to examine entire storm events to reveal the spatial structure of windstorms, rather than viewing local station gust time-series independently of each other. We define the storm footprint as the collection of local maximum gusts over the entire duration of a synoptic storm. The strong correlation between peak gust and risk of damage ensures the storm footprint is appropriate for risk assessment. The creation of the set of 135 storm footprints is now described.

An algorithm identified the days on which peak gusts from extra-tropical windstorms were potentially damaging. Extratropical windstorms were identified by applying two main constraints: first, the storms must occur between September and May (thereby excluding local convective events in summer) and second, at least ten stations in the domain must suffer gusts in excess of $20 \mathrm{~m} \mathrm{~s}^{-1}$. This windspeed corresponds to a threshold above which damage to buildings is likely to occur and ten stations ensure that small-scale events are excluded. This algorithm identified 2720 days with potentially damaging winds in the European domain consisting of 15 countries in the last $38 \mathrm{yr}$.

A daily peak gust footprint was created for each of these 2720 days and put into Risk Management Solutions European Windstorm 2011 (RMS EUWS 2011) catastrophe loss model. The creation of the daily peak gust footprint is described in Cusack (2012) and only brief details are now given. The station peak gust data are quality controlled using a variety of information to ensure the representativeness of the station with respect to the surrounding area, then interpolated to a Variable Resolution Grid (VRG) with resolution ranging from 1 to $10 \mathrm{~km}$, using Barnes interpolation (Barnes, 1964) with modifications to suit the unique spatial character of peak gusts.

The set of 135 storm footprints were selected from the super-set of (potentially damaging) 2720 daily footprints by selecting the dates of the top $N$ damaging storms in each of the 15 countries, where $N$ is about 20 to 25 for major countries such as France, Germany, and the UK, about 20 for second tier countries including Austria, Benelux, and the Scandinavian countries, and about 10 to 15 for smaller countries subject to smaller windstorm damage such as the Czech Republic and Slovakia. A storm footprint is distinct from daily footprints. This is because a single cyclone may cause damage on consecutive days as it traverses the domain. Each footprint in the set of 135 storms used various sources of information to identify the peak gust of a storm, rather than the daily peak gust, including: (i) daily peak gust time-series at stations, (ii) NCEP re-analyses of surface pressure, (iii) DWD operational analyses of surface pressure and (iv) reports of damage in the public domain such as media, internet. Figure 2 contains an example of a storm footprint for Daria, a major windstorm event in January 1990.

Finally, an additional aggregation from VRG to a coarser resolution, known as CRESTA, was performed. CRESTA zones are defined with respect to the distribution of population and buildings and cover areas that, in most countries, correspond to 2 digit postal codes. At this resolution 996 cells cover the entire domain of the 15 countries. Figure 1 displays the shapes of these CRESTA cells. This coarser resolution is more similar to the weather station data (at about $50 \mathrm{~km}$ resolution) than the VRG with cell sizes of 1 to $10 \mathrm{~km}$.

\section{Model methodology}

\subsection{Extreme value copulas}

We begin this section with a general overview of copulas, then give a formal description of the extreme value copulas that we have used to capture the spatial dependencies of $\mathrm{Eu}-$ ropean windstorms.

Multivariate distributions of damaging wind at two or more distinct locations can be thought of in terms of the marginal distributions of winds and their mutual dependence. The use of parametric models to define the marginals and their dependence can be beneficial for data analysis since (1) the parametric models usually condense the multivariate information into a few parameters, (2) if extra evidence indicates such models are good approximations of the processes generating the data, then the parametric models could be used for quality control of the data, and (3) these parameters can provide information on all expected outcomes, rather than the truncated view provided by finite-length datasets.

Multivariate distributions may consist of marginals with large differences in magnitude which can complicate the dependency structure between random variables. A preprocessing step to convert the marginals to values from 0 to 1 using their respective cumulative distribution functions creates uniform marginals for all random variables and standardizes the form of the dependence structure. A copula is the multivariate distribution describing the dependence structure between such uniform marginals. Different classes of copula functions have been found to suit particular types of multivariate data. These copulas often have only one unknown parameter, simplifying both the fitting and the interpretation.

The first step in the copula-based method is to specify the form of the marginal distributions. We adopt the standard peak-over-threshold (POT) theoretical model and assume all marginal distributions to be Generalized-Pareto Distributions (Coles, 2001). The effect of using a subset of 135 storm events in the full storm catalogue upon the fitting of the marginal distributions was investigated. By construction, the set of 135 historical storms is incomplete for gust levels with a Return Period (RP) below $1 \mathrm{yr}$ in the central part of the domain and $2 \mathrm{yr}$ in rural and peripheral areas. In the context of fitting a GPD marginal to locations, the set of 135 storms acts as a threshold selection due to its truncation of the full historical catalogue of storms. We determined the validity of 
the GPD fits based on 135 storms by assessing the independence of shape and scale parameters upon the threshold parameter of the GPD (Coles, 2001; Della-Marta et al., 2008). This was tested using the full catalogue of 2720 stormy days. The minimum gust resolved by the 135 storms falls in a region of acceptable threshold values for determining shape and scale parameters. Therefore, the choice of using a subset of 135 storms had no adverse impacts on accuracy of fitting marginal distributions, and contained the benefit of more intense quality assurance of these more major storms.

The copula function, defined as the joint distribution of two or more random variables based on their uniform marginal distributions, is now described more formally. A copula $C(u)=C\left(u_{1}, \ldots, u_{n}\right)$ is a $n$-dimensional joint distribution defined on $[0,1]^{n}$ with standard uniform marginals. The mapping $C:[0,1]^{n} \rightarrow[0,1]$ is a $\mathrm{n}$-dimensional copula if $C\left(u_{1}, \ldots, u_{n}\right)$ is increasing in each component $u_{i}$, the marginals are uniform and the rectangle inequality holds Nelsen (1999); Marshall (1996); Hutchinson and Lai (1990).

Sklar's Theorem states that for any joint distribution function $F$ for $n$ variables, and respective marginal distributions, there exists a copula $C:[0,1]^{n} \rightarrow[0,1]$ such that:

$F\left(x_{1}, \ldots, x_{n}\right)=C\left(F_{1}\left(x_{1}\right), \ldots, F_{n}\left(x_{n}\right)\right)$

In other words, the probabilities $F$ can be derived either directly from the joint distribution function or via the marginal distributions and a copula. We are here interested in copula families that are suitable for representing the behavior of multivariate maxima, i.e. Multivariate Extreme Value (MEV) copulas. Galambos (1987) was the first to formalize the theory of multivariate maxima as a copula theory. We encourage the interested reader to refer to chapter 7 of McNeil et al. (2005) for a clear introduction to extreme value copulas.

One particular procedure for constructing extreme value copulas was proposed by Pickands (1981). In the bivariate case, an extreme value distribution is written using the general representation:

$C(x, y)=\exp \left(\ln u_{1}+\ln u_{2}\right) A\left(\frac{\ln u_{2}}{\ln u_{1}+\ln u_{2}}\right)$,

where $u_{1}=F_{X}(x), u_{2}=F_{Y}(y)$ are the marginal distributions and $A$ is the so-called Pickands dependence function. Pickands representations are convex on $[0,1]$. The marginal components are independent if and only if $A(w)=1$ for all $w=\ln u_{2} /\left(\ln u_{1}+\ln u_{2}\right) \in[0,1]$. The components are completely dependent if $A(w)=\max (w, 1-w)$ for all $w \in[0,1]$. Any convex differential function satisfying these conditions can be used to construct extreme value (EV) copulas.

Given the censored nature of our dataset, we need to model multivariate threshold exceedances. We assume that the vectors $\boldsymbol{X}_{1}, \ldots, \boldsymbol{X} n$ have unknown joint distribution $F(\boldsymbol{x})=C\left(F_{1}\left(x_{1}\right), \ldots, F_{n}\left(x_{n}\right)\right)$ for copula $C$ and marginals $F_{1}, \ldots, F_{n}$. We are interested in the upper tail of $F(x)$ above a threshold vectors $\boldsymbol{t}=\left(t_{1}, \ldots, t_{n}\right)^{\prime}$. In the univariate case we have that for $x_{j}>=t_{j}$ and $t_{j}$ high enough, the tail of the marginal distribution $F_{j}$ may be approximated by a Generalized Pareto distribution (GPD, Coles, 2001):

$F_{j}\left(x_{j}\right) \approx \tilde{F}_{j}\left(x_{j}\right)=1-\lambda_{j}\left(1+\xi_{j} \frac{x_{j}-t_{j}}{\beta_{j}}\right)^{1 / \xi_{j}}$,

where $\lambda_{j}=\tilde{F}_{j}\left(t_{j}\right), \xi_{j} \neq 0$ is the shape parameter, and $\beta_{j}$ is the scale parameter.

A heuristic argument suggests that a similar approach should be viable for multivariate threshold exceedances. It has been demonstrated by Reskin (1987) that for $\boldsymbol{x} \geq \boldsymbol{t}$ we can use the approximation:

$F(\boldsymbol{x}) \approx \tilde{F}(\boldsymbol{x})=C_{0}\left(\tilde{F}\left(x_{1}\right), \ldots, \tilde{F}_{n}\left(x_{n}\right)\right)$,

where $C$ has been replaced by its limiting EV copula $C_{0}$. In other words, we can model the probabilities $F$ using the GPD models for the marginals, and $C_{0}$ for the copula. In line with many examples of this model found in the literature, we use the Gumbel copula family as the limiting EV copula $C_{0}$ (Steinkohl et al., 2010; McNeil et al., 2005). The Pickands function for the Gumbel copula is:

$A(w)=\left(w^{r}+(1-w)^{r}\right)^{1 / r}$

where $r$ is the dependence parameter. The bi-variate Gumbel EV-copula is then completely described by Eqs. (2) and (5).

\subsection{Statistical model fitting}

We use censored likelihood (Ledford and Tawn, 1996) to fit the copula and marginal distribution parameters. In the bivariate case, the overall likelihood is the product of 4 partial likelihoods that account for both components being above or below thresholds, or one component above and the other below. We compute copulas for all pair combinations of 996 CRESTA cells where at least 10 data points are above the threshold. The marginal distributions are assumed to be GPD.

Goodness of Fit (GOF) tests for multivariate distributions is an active field of research, and several methods have been proposed. Schölzel and Friederichs (2008) provided a useful summary of the ongoing research literature. Multidimensional chi-square tests as given in Fermanian (2005) are easy to apply but require probability binning and there is no optimal choice of bin width and number. More refined GOF tests are based on the Probability Transform Integral and project the multivariate problem into a univariate distribution allowing standard chi-square tests to be applied. These methods were initially proposed by Breymann et al. (2003) and further refined by Berg and Bakken (2005).

A natural choice for our analysis is the goodness of fit test proposed by Genest et al. (2011) for bivariate extreme-value copulas. Under the assumption that $C$ is an extreme value copula, we aim to test the null hypothesis that $A$ belongs to a specific parametric class, in our case the Gumbel copula 

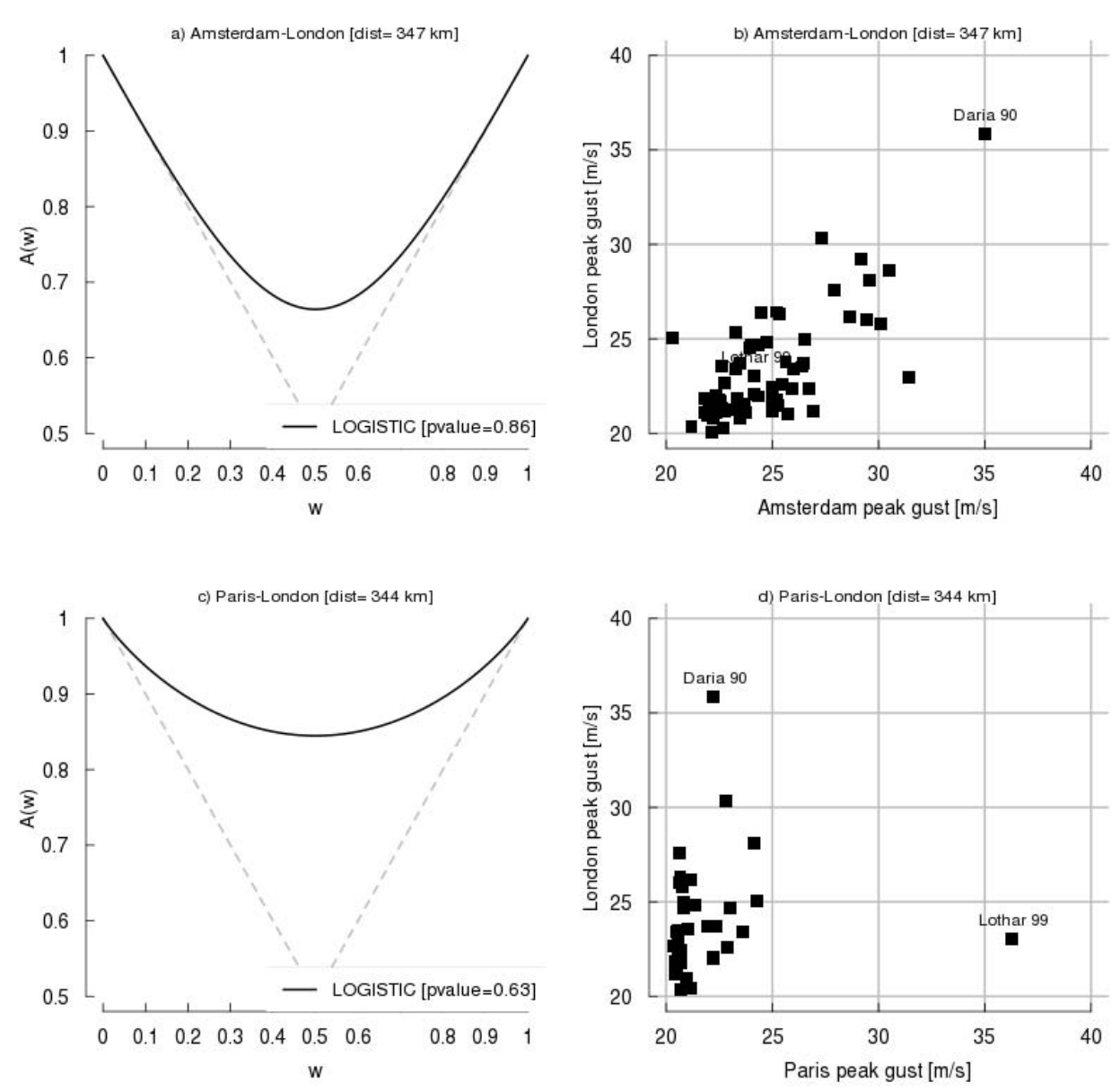

Fig. 3. Pickands functions and scatter plots of [3]s peak gust from historical reconstruction footprints for location pairs London-Amsterdam and London-Paris.

family. The test is based on the Cramèr-von Mises statistic that measures the distance between an estimate of the parametric Pickand dependence function and the non-parametric Pickands estimators proposed by Genest and Segers (2009). The test is estimated via a parametric bootstrap procedure (Yan, 2007; Kojadinovic and Yan, 2007; R Development Core Team, 2011). We retain all copulas for which the null hypothesis can not be rejected with a confidence level of $95 \%$.

The Pickands functions for the pairs Amsterdam-London and Paris-London, together with gust scatter-plots, are shown in Fig. 3. The record of wind gusts at Amsterdam-London shows a higher degree of dependence than London-Paris due to the typical zonal direction of storm tracks. Correspondingly, the Amsterdam-London Pickands function is closer to the theoretical minimum $A=\max (w, 1-w)$ for complete dependence (dashed gray line) than the London-Paris Pickands function.

\subsection{Tail dependence}

Scalar measures can be useful in providing a higher-level and more intuitive view of pairwise dependence than the Pickands function. There are various scalar measures of spatial dependence, such as linear correlation, rank correlation and the coefficient of tail dependence. Most meteorological and climate data belong to non-Gaussian skewed or bounded distributions (e.g. Schölzel and Friederichs, 2008; Wilks, 2005) and so linear correlation coefficients are inappropriate (referred to as Fallacy 1 in McNeil et al., 2005). Low linear correlation between the values at two locations of a nonGaussian meteorological variable should not be interpreted as a proof of independence. Rank correlation and tail dependence are more general copula-based measures suitable for the parametrization of the non-elliptic multivariate distributions occurring in meteorology.

The tail dependence coefficient provides a scalar measure of the dependence between two locations in the tail of a bivariate distribution. This index was developed by Geffroy (1958) and Sibuya (1960). Falk et al. (2000) provides an in 
depth description of its properties. Steinkohl et al. (2010) applied this measure to analyze the dependence structure of wind speeds at various meteorological masts in Denmark.

The tail dependence coefficient $\chi$ is the conditional probability of one variable being extreme given that the other is extreme:

$\chi=\lim _{t \rightarrow \infty} P\left(F_{X}(X)>t \mid F_{Y}(Y)>t\right)$

It can be shown that $\chi$ is related to the Pickands representation $A$ - defined for the Gumbel EV-Copula in Eq. (5) by:

$\chi=2\left(1-A\left(\frac{1}{2}\right)\right)$

$\chi$ is a probability ranging between 0 (independent copula) and 1 (comonotonicity copula). As an aside, note that the coefficient of tail dependence of the Gaussian copula is always 0 regardless of the value of the correlation coefficient, hence, the Gauss copula is asymptotically independent in both tails.

The estimated $\chi$ values for the pairs Amsterdam-London and Paris-London, shown in Fig. 3, are 0.67 and 0.31, respectively. In other words, if an extreme peak gust from an extra-tropical cyclone is observed in London, there is a probability of 0.67 that an extreme peak gust value will be recorded in Amsterdam, and only a 0.31 probability that this would happen in Paris. The spatial structure of tail dependence is of obvious importance for re/insurance applications. The greater the spatial extent of this dependence in the domain, the greater the aggregated damaging hazard from individual storm events, which will tend to produce thicker tails of storm risk distributions.

\section{Results}

The spatial structure of European windstorms is now explored in greater detail. Section 4.1 describes the spatial structure of the tail dependence in the domain, with emphasis on some of the major cities. Section 4.2 assesses the variability of the windstorm tail dependence structure with a major mode of climate variability in Europe often used to characterize climate change. Finally, results from an alternative and simpler method of charactertizing the gust dependence structure are compared to those from our copula method.

\subsection{Spatial structure of tail dependence}

The tail dependence coefficient (defined as the probability of observing an extreme gust at one location, given that an extreme gust is observed at the second location, see Eq. 6), was estimated for each combination of pairs of the 996 CRESTA cells in the 15 countries in our domain. It was found that tail dependence is generally a function of distance between centroids of CRESTA cells, see Fig. 4. The tail dependence is consistently high up to a CRESTA separation distance of

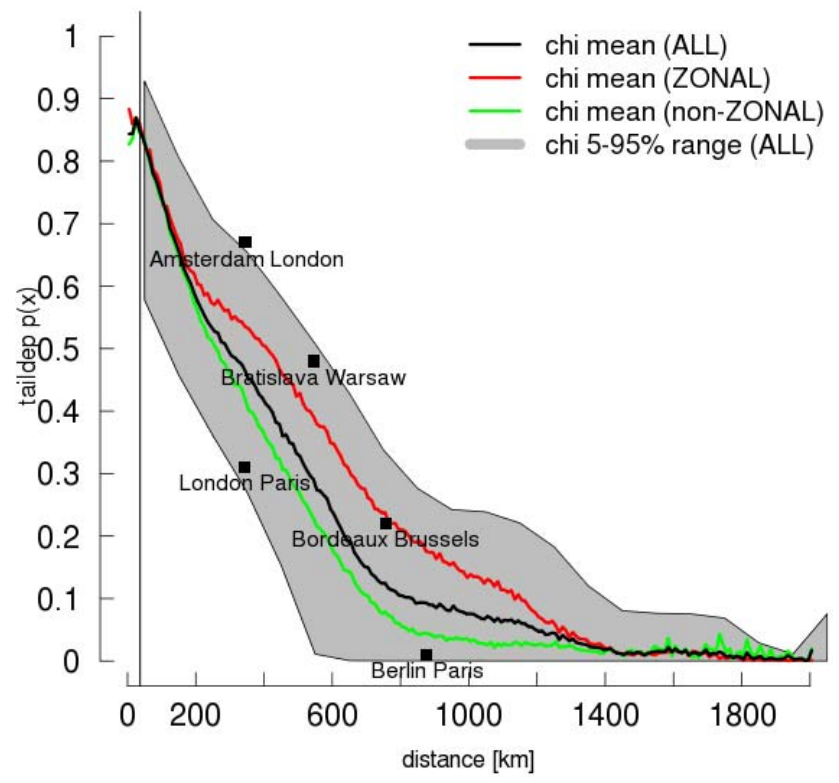

Fig. 4. Tail dependence $\chi$ as a function of distance between CRESTA centroids and orientation. Zonal CRESTA pairs are defined to have bearing angle between 90 and 120 degrees. Black vertical line (shown at $x=30 \mathrm{~km}$ ) shows mean CRESTA grid spacing.

500km: the average $\chi$ value is 0.49 . This illustrates the importance of capturing the right spatial correlation structure for extra-tropical storms. On average over all pairs of locations, we find that any two locations within a radius of $500 \mathrm{~km}$ have probability 0.49 of experiencing extreme gusts during the passage of a singe extra-tropical storm given that one of the locations is hit. Figure 5 shows four maps of tail dependence coefficients centred in London, Copenhagen, Paris and Berlin. These maps depict the probability of observing extreme gusts at any location in Europe given that one of these four cities is hit; $\chi$ equals 1 at the location where we centered the plot, and slowly decreases as the separation distance increases. These four cities have a dependence structure preferentially orientated along the zonal direction of the main storm track system.

The directional dependence of this spatial structure was quantified by specifying a zonal subset of CRESTA pairs for which the eastern CRESTA is situated between 60 and 120 degrees of the western CRESTA. It was found that the zonal tail dependence extends further than in meridional tail dependence, see Fig. 4. At a CRESTA separation distance of $500-1000 \mathrm{~km}$, the probability $\chi$ is 0.15 when averaged over all copulas, and rises to 0.26 for pairs of CRESTAs in the same latitude zone: the probability of joint extreme events almost doubles in the zonal direction.

This spatial anisotropy of the tail dependence contributes significantly to the behavior of joint wind gusts experienced at specific locations. CRESTA cells were selected for each of 

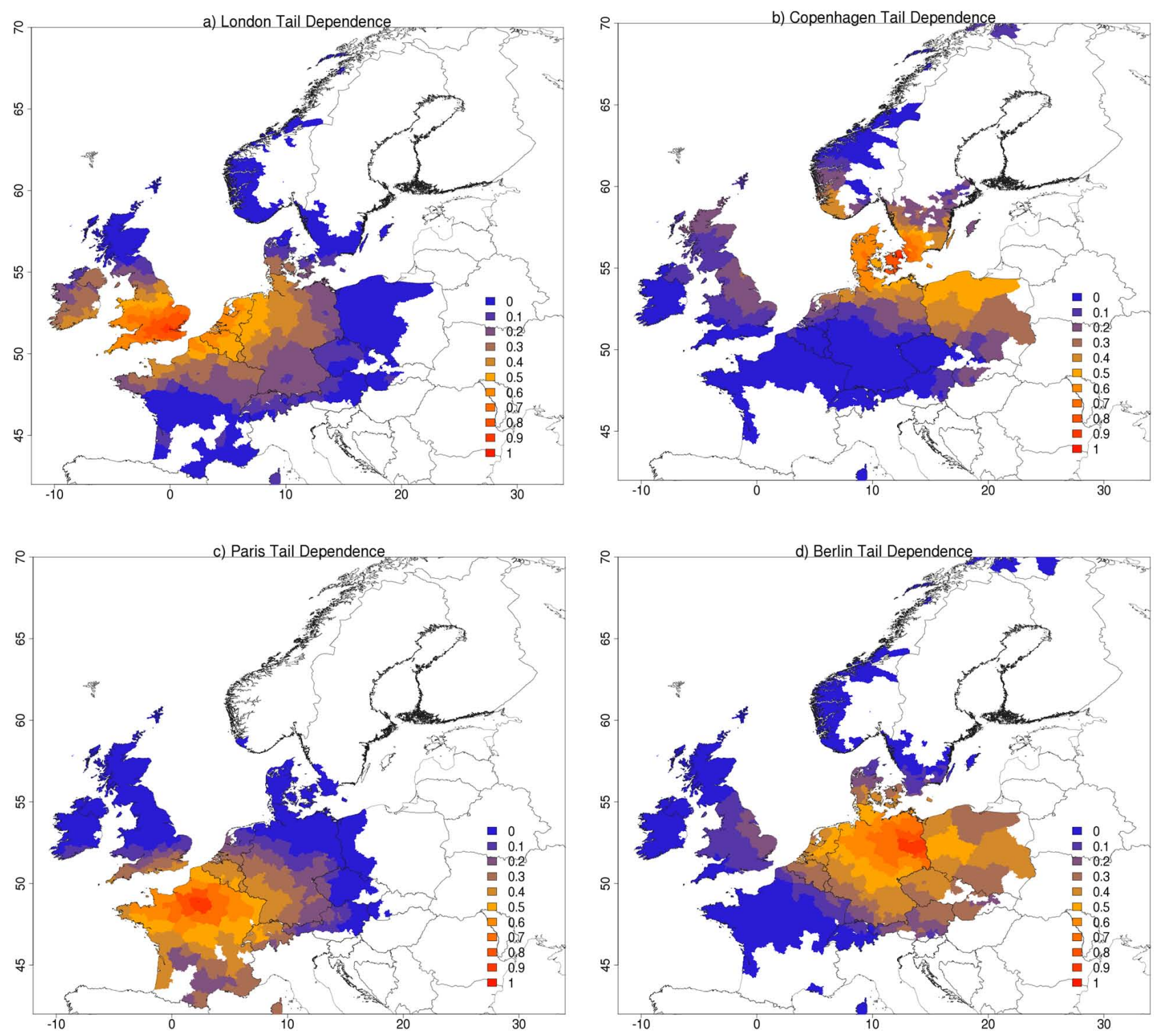

Fig. 5. Maps of tail dependence coefficients $\chi$, centered in London (a), Copenhagen (b), Paris (c), and Berlin (d). Each map shows $\chi$-values between selected cities and all other CRESTAs in the domain.

22 major European cities; Amsterdam, Berlin, Copenhagen, Hamburg, London, Munich, Paris, Bordeaux, Dublin, Edinburgh, Frankfurt, Lyon, Manchester, Stockholm, Vienna, Zurich, Oslo, Bratislava, Prague, Warsaw, Luxembourg and Brussels. Figure 6 is a visualization of the pairwise relations using cities as vertices and tail-dependence as edge strength with cut-off value $\chi=0.4$.

All vertices are connected by at least one edge with the exclusion of Oslo and Stockholm. Scandinavian cities lie on the edges of the graph. The three major extra-tropical storms in the historical record for Denmark, Sweden and Norway are respectively Anatol, Erwin and Nyttarsdag which were mostly confined to the Scandinavian region. Based on an integral measure of storm intensity and area (see Eq. 7) Nyt- tarsdag has been the most intense hazard event in Norway and is the 45th in Sweden and 53rd in the UK. Anatol ranks as the first and second most intense storm in Denmark and Sweden, the 10th in Poland and 32nd in the Netherlands. Erwin has been the most intense storm in Sweden, the second in Denmark and the 12th in the UK.

On the other hand, cities such as Amsterdam, Brussels, Frankfurt and Hamburg, closer to the center of the domain, are found in the core of the graph. Key drivers of this wide region of highly inter-correlated vertices are storms in the 1990s. Storm Daria ranks as the first or second most intense national event in the UK, Germany, Belgium, and Netherlands. Storm Vivian ranks in the top four most intense national events in the UK, Germany, Denmark, Belgium, 


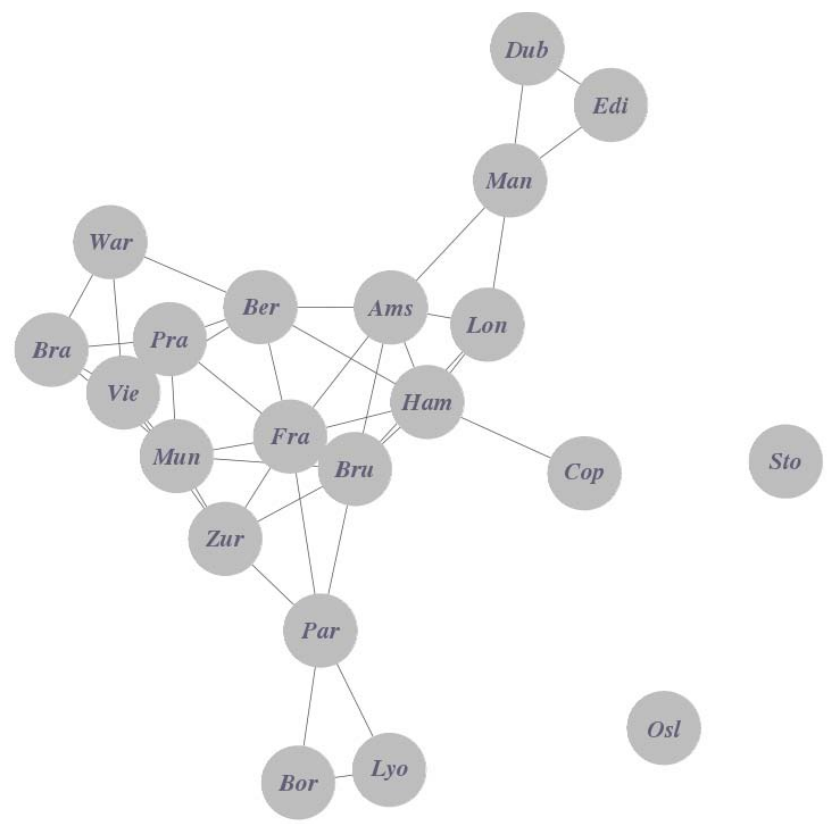

Fig. 6. The graph synthesizes the strongest tail dependences between European capitals. Each circle (vertex) represents a city and connections are drawn for pair of cities whose tail dependence is bigger than cut off value $\chi=0.4$. The graph can also be interpreted as a representation of the triangular matrix of tail dependence coefficients between pairs of cities. Graph orientation is randomly chosen.

Switzerland, Luxemburg, Ireland, and the Czech Republic. London and Paris, with $\chi=0.31$, are not linked in the graph. An in-depth analysis of the London-Paris dependence structure is presented in Sect. 4.2.

East European cities such as Warsaw, Vienna and Prague are linked to German cities that lie in the middle of a large region of correlations, extending both eastward and westward of these German cities.

\subsection{Benchmarking catalogues of storms}

As mentioned in the Introduction, a catastrophe model contains a stochastic set of events that expands the time-frame of the historical catalogue to thousands of years. Multi-variate distributions are readily extracted from these stochastic catalogues using empirical Cumulative Distribution Functions (CDFs). There is a need to ensure that the synthetic storms of a catastrophe model are rooted in historical, observed behavior. We show here how the copula-based analysis of historical storms can be used to define benchmarks of storm spatial structure, for validation of this important aspect of catastrophe models.

The maps of tail dependence coefficient provide conditional probabilities of gust exceedance between one point and all others in the model domain. Here we choose to view one pair of locations, for many different gust exceedance values.
For benchmarking purposes, we define targets as univariate functions of joint distributions of wind gust at two different locations. Minimum, maximum and mean gust at any two locations are possible choices for the univariate function. The maximum gust does not guarantee damage at both locations and is therefore not appropriate. The mean gust is a potentially misleading indicator of joint damage, given the exponential nature of damage functions. For these reasons, we concentrate on the minimum gust (MG).

The simplification of considering scalar functions allows univariate extreme distributions to be fitted. The tail of a random variable defined as the scalar function of multivariate random variables can be generally approximated by a GPD (Leadbetter and Rootzen, 1988). Hence two methods can be used to estimate the distribution of true MG; (i) fit a univariate GPD function; (ii) fit extreme value copulas.

Here we focus on the pairs London-Paris and Berlin-Paris to provide an example of this methodology. London and Paris experienced exceptional gusts (and corresponding damage) during storms Daria (1990) and Lothar (1999), respectively, with measurements showing gust wind speed well above $35 \mathrm{~m} \mathrm{~s}^{-1}$ in city stations. However, there is no record of gusts above $25 \mathrm{~m} \mathrm{~s}^{-1}$ at the two locations during the passage of a single windstorm: storms Daria and Lothar were the most damaging storms in Europe for decades but neither of the two storms was felt strongly in both Paris and London. Based on our historical reconstructions of windstorm footprints, the largest MG in London and Paris was recorded during the passage of storm Vivian in 1990. Panel (a) of Fig. 7 shows the exceedance probability of MG between Paris and London for both methods, together with the marginal gust distributions at each site. Whilst the univariate and copula targets agree at return periods (RP) of $10 \mathrm{yr}$ and shorter, they diverge at longer RPs. The univariate GPD matches the observed MG from RP10 to 40, whilst the copula-based estimate follows an exponential pattern that is similar to the slope of the Paris marginal gust distribution. As a consequence, the GPD-based estimate is $5 \mathrm{~m} \mathrm{~s}^{-1}$ lower than EVCopula target at RP200 (panel (a) of Fig. 7).

On the other hand, MG targets for Berlin-Paris derived from univariate GPD and EV-Copulas are substantially in agreement (panel (b) of Fig. 7). At RP200 both target MGs are just below $26 \mathrm{~m} \mathrm{~s}^{-1}$. The exponentially-shaped marginal distribution of gust in Paris does not translate into a fat-tailed MG distribution because the Paris-Berlin tail dependence coefficient is 0.01 , i.e. the two distributions are close to tail independence.

London-Paris and Berlin-Paris have experienced comparable MGs over the last $40 \mathrm{yr}$, but London-Paris observations show an higher degree of dependence than Paris-Berlin, which is mainly driven by a cloud of medium-intensity storms hitting Paris and London with similar intensities. In fact rank correlations for London-Paris and Berlin-Paris are 0.36 and 0.03 respectively. The univariate GPD method can not discriminate between these pairs of locations on the basis 

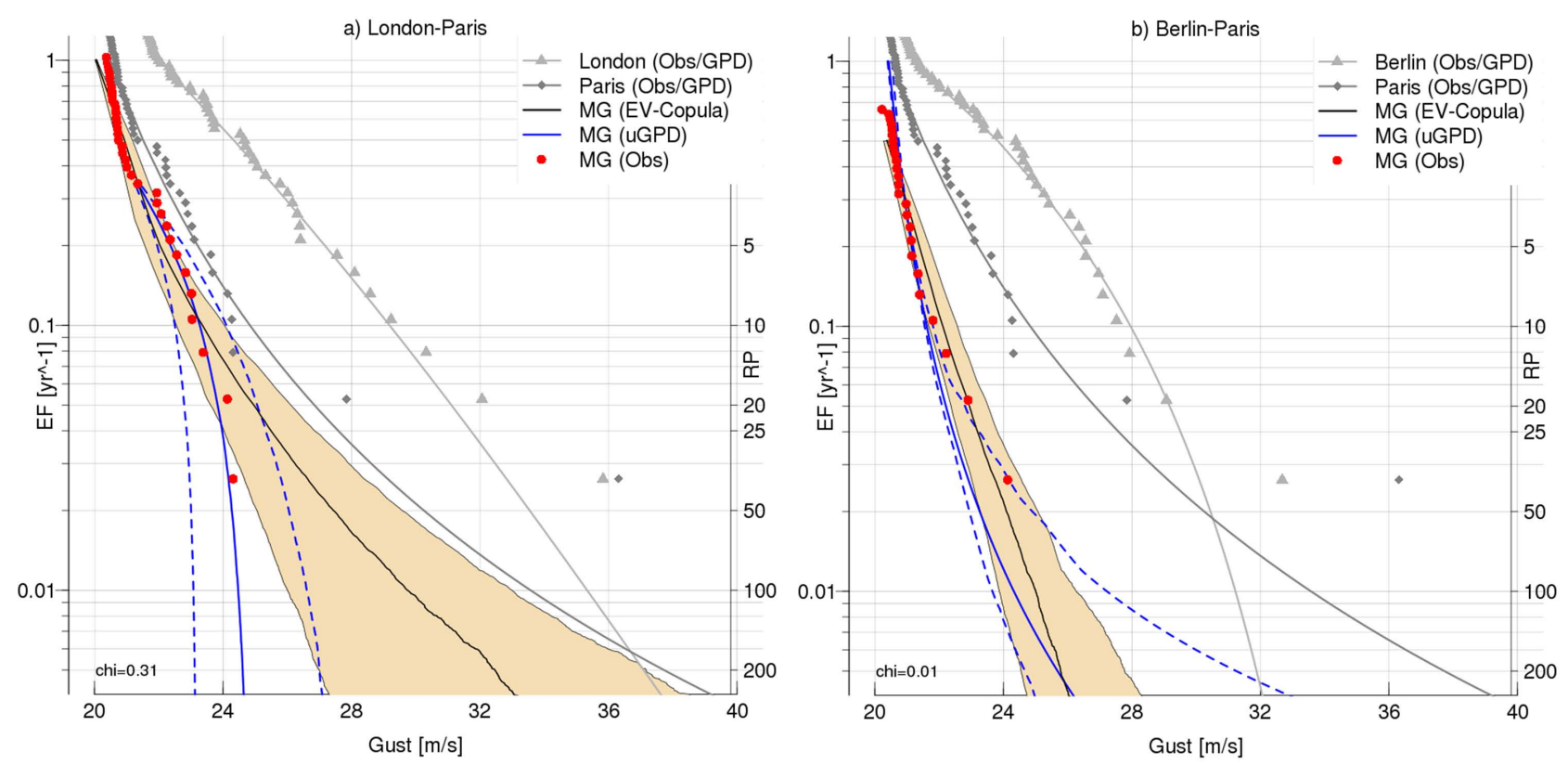

Fig. 7. Calibration targets for London-Paris (a) and Berlin-Paris (b). Gray lines and dots show marginal gust Exceedance Frequencies (EF [yr ${ }^{-1}$ ) and observed gust values at each location separately. Red dots show observed M(inumum) G(usts) exceeded at the two locations during the passage of a single storm. Solid blue lines depict targets as derived from univariate GPD fiting on observed MGs (dashed blue lines represent $95 \%$ confidence level), while black solid lines show EV-Copula targets (95\% confidence level is shown in yellow). Note that EV-Copula and univariate GPD targets differ for London-Paris and are in much closer agreement for Berlin-Paris.

of historical MG values and produce similar estimates of MG at all RPs. The EV-Copula method factors in the strength of tail dependence which results in clear separation of MG estimates for RPs longer than $10 \mathrm{yr}$ for London-Paris and BerlinParis.

\subsection{Sensitivity to climate variability}

Stochastic catalogues of storms are usually based on the longest possible historical climatology with no regard to climate variability and possible future trends. In this section, the sensitivity of aggregated damaging hazard to climate variability in Europe is addressed. The mechanisms of change will be assigned to the components of the joint distribution, namely the storm frequency, marginals and storm dependence structure.

The North Atlantic Oscillation (NAO) is the major mode of inter-annual variability in the winter-time in the north Atlantic basin (Wallace and Gutzler, 1981) and modulates the atmosphere and surface climate characteristics in northern and central Europe through variations in orientation and strength of the Atlantic jet-stream (Kushnir, 2006). The NAO has been found to modulate the variability of extreme storms in the Atlantic and European sector via these changes to the jet-stream, e.g. Cusack (2012). We therefore explore sensitivity of joint damaging hazard to changes in the phase of the NAO.
To identify a signal of NAO modulation on damaging wind storms, we separated the 135 historical reconstructions into two groups according to the phase of the monthly NAO index published by NOAA (http://www.cpc.ncep.noaa.gov). This assigns $20 \mathrm{yr}$ to the positive NAO phase (NAO+) and 18 to the negative NAO phase (NAO-). To counteract the unwelcome effects of small sample sizes (and hence larger uncertainties) caused by splitting the data into two groups, the CRESTA level data have been aggregated into a regional Storm Severity Index (SSI) defined as:

$\mathrm{SSI}=\left(\frac{\sum\left(v_{i}-v_{0}\right)^{3} \cdot A_{i}}{\sum A_{i}}\right)^{1 / 3}$

where $v_{i}$ is the wind gust at cell $i, A_{i}$ is the area of the $i$-th CRESTA cell, $v_{0}$ is a threshold set to $20 \mathrm{~m} \mathrm{~s}^{-1}$, and the summation is over all CRESTA cells in the studied region. The SSI is a hazard-based index which correlates closely to aggregated damages due to storms. It has constituted the basis of the first European windstorm parametric index Cat Bond undertaken by RMS and issued in 2000. There are other measures of storm intensity based solely on winds (e.g. Lamb, 1991; Klawa and Ulbrich, 2003; Leckebusch et al., 2008); however, the index defined in Eq. (7) has been chosen for its relevance to the re/insurance industry.

The SSI is defined for four regions with storm variability governed by the NAO (Mailier et al., 2005): UK south of $53^{\circ} \mathrm{N}$ (south-UK), France north of $48^{\circ} \mathrm{N}$ (north-FR), 

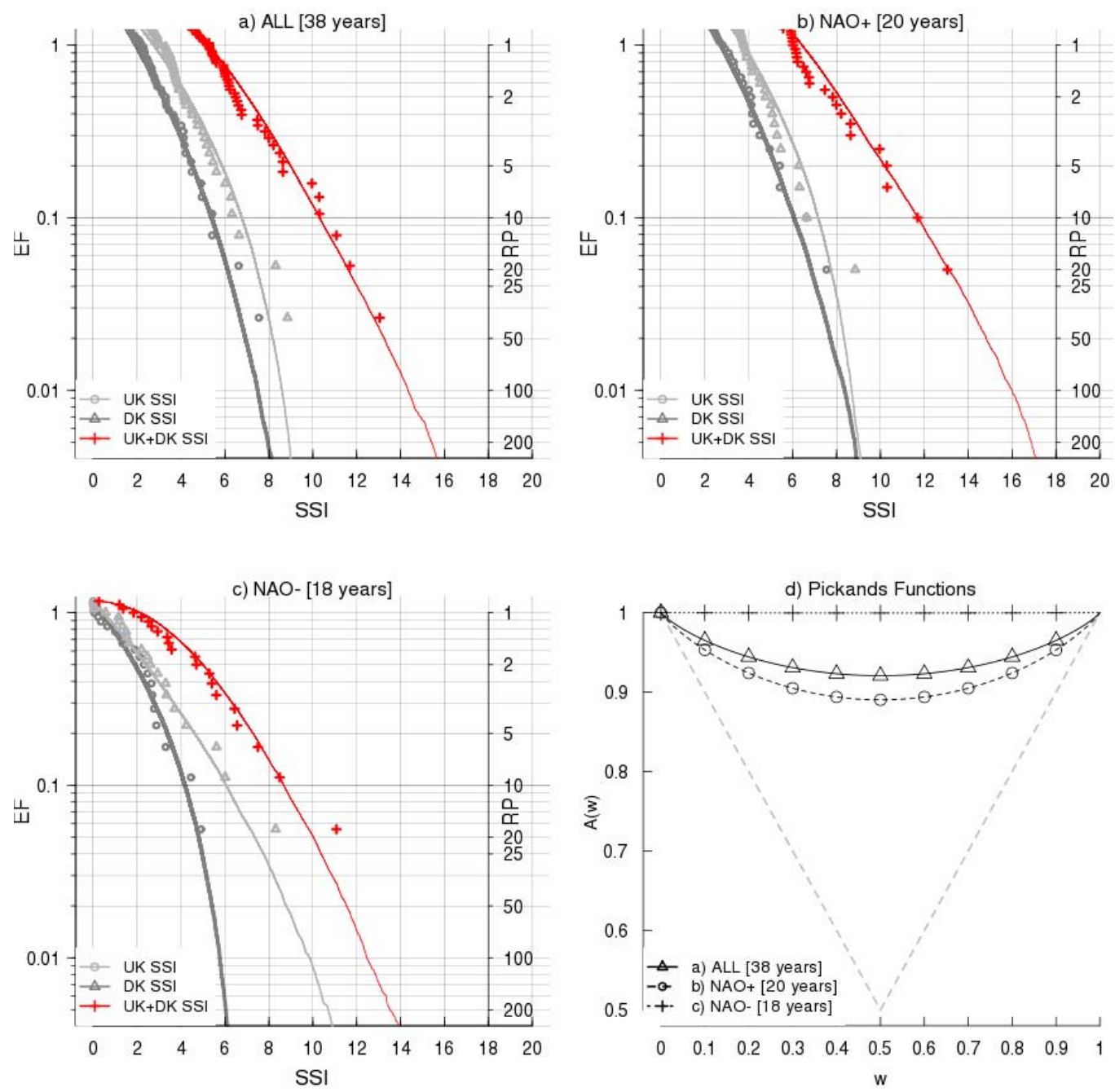

Fig. 8. SSI event Exceedance Frequency (EF) for the regions south-UK, west-DK and south-UK + west-DK. Dots/Triangles/Crosses are observed values, while solid lines are estimates based on GPD (south-UK, west-DK) or EV Copula (south-UK + west-DK). Panel (d) depicts the Pickands function for south-UK - west-DK copulas for the three cases considered; no extremal dependence is evident during the negative NAO phase (NAO-), while a weak dependence structure emerges during the positive NAO phase (NAO+).

Germany north of $51^{\circ} \mathrm{N}$ (north-DE), and Denmark west of $14^{\circ} \mathrm{E}$ (west-DK). Bivariate EV-copulas are fit to pairs of regions for both the NAO+ and NAO- subsets of data, and for the entire record of historical wind storm events. We present results in terms of expected frequency of events above threshold and we refer to RP, in a rather loose sense, as the inverse of frequency. We estimate the RPs of the sum of SSI over a pair of regions from random samples drawn from EVcopulas; the SSI RPs for each region are derived from random samples of marginal distributions.

Figure 8 shows the RPs of SSI in region south-UK, westDK and for the sum of SSI in south-UK + west-DK for negative and positive NAO phases, and the full record. Extratropical storms are more likely to happen during positive phase of NAO- [64]\% of events used in this analysis occurred during NAO+ months - and we consistently estimate higher SSI values at all return period for the NAO+ set. Figure 8 indicates that EV-copulas are good estimators of the distribution of sum of SSI and can be used to estimate the conditional probability of extremes.

Over the 6 combinations of pairs from 4 regions, only two pairs (south-UK + west-DK, and south-UK + north-FR) show a variation greater than $10 \%(+18$ and $+10 \%$, respectively) in the strength of tail dependence between $\mathrm{NAO}+$ and NAO-. Region (west-DK, south-UK) shows no extremal dependence during negative NAO but a weak dependence structure emerges during its opposite phase, see Fig. 8. 


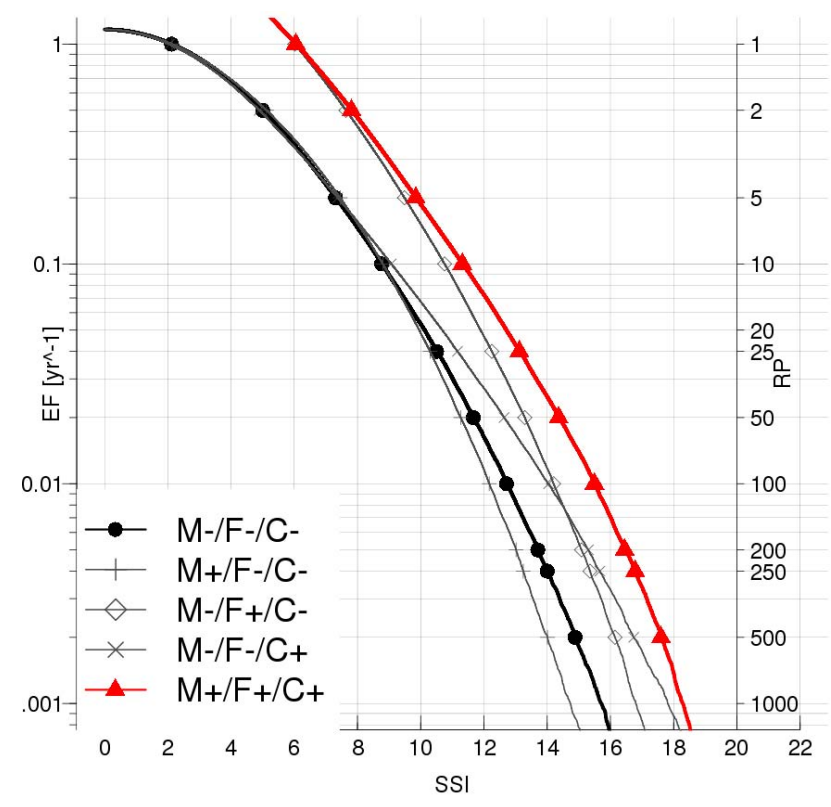

Fig. 9. $\mathrm{M}$ (arginal), F(requency) and $\mathrm{C}$ (opula) contribution to south$\mathrm{UK}+$ west-DK SSI EEF changes due to NAO phase (+ positive/negative). $\mathrm{M}+/ \mathrm{F}+/ \mathrm{C}+$ and $\mathrm{M}-/ \mathrm{F}-/ \mathrm{C}-$ have been fitted to data and are identical to red lines in panel $\mathrm{b}$ and $\mathrm{c}$ of Fig. 8, while $\mathrm{M}-/ \mathrm{F}+/ \mathrm{C}+$, $\mathrm{M}+/ \mathrm{F}-/ \mathrm{C}+$ and $\mathrm{M}-/ \mathrm{F}-/ \mathrm{C}+$ are synthetic Copulas constructed using NAO-components except one. Frequency distribution is the single most important driver of SSI EEF change going from NAOto NAO+ up to RP $200 \mathrm{yr}$. For longer RPs the increase of tail dependence observed during positive NAO phase plays a comparable role.

An experiment was run to analyze the changes in the sum of SSIs in regions south-UK and west-DK according to NAO phase.

We separated the parameters of the Gumbel EV-copula distributions in 3 groups: the frequency for storm occurrence (F), the scale and shape parameters of the marginal GPDs (M) and the dependence parameter of the Pickands function (C). For $\mathrm{NAO}+$ phase we estimated parameters $\mathrm{M}+, \mathrm{F}+$ and $\mathrm{C}+$. Similarly for NAO- years we estimated parameters $\mathrm{M}-, \mathrm{F}-$ and $\mathrm{C}-$.

Once the F, $\mathrm{M}$ and $\mathrm{C}$ parameters were known, the total sum of SSI in the two regions could be estimated by sampling from the joint distribution. In addition to positive and negative NAO Copulas - hereafter $\mathrm{M}+/ \mathrm{F}+/ \mathrm{C}+$ and $\mathrm{M}-/ \mathrm{F}-/ \mathrm{C}-$ - three Copulas were created, replacing one component of $\mathrm{M}-/ \mathrm{F}-/ \mathrm{C}-$ with its positive counterpart. The synthetic Copulas $\mathrm{M}+/ \mathrm{F}-/ \mathrm{C}-, \mathrm{M}-/ \mathrm{F}+/ \mathrm{C}-$ and $\mathrm{M}-/ \mathrm{F}-/ \mathrm{C}+$ were then used to assess the relative importance of each $\mathrm{NAO}+$ component as shown in Fig. 9.

The difference in sum of SSI due to changes in frequency (3.35 events per year during $\mathrm{NAO}+$, and 1.16 events per year during $\mathrm{NAO}-$, based on comparing $\mathrm{M}+/ \mathrm{F}-/ \mathrm{C}-$ with $\mathrm{M}-/ \mathrm{F}-/ \mathrm{C}-)$ is the dominant contributor to total changes in the sum of SSI between NAO+ and NAO- years up to
RP 200 yr. At longer RPs the $18 \%$ increase in the strength of tail dependence (based on comparing $\mathrm{M}-/ \mathrm{F}-/ \mathrm{C}+$ with $\mathrm{M}-/ \mathrm{F}-/ \mathrm{C}-$ ) plays a similar role to the other characteristics in determining the overall change.

This result suggests that a relatively minor change in the dependence structure can be more important than a major change in the frequency distribution in terms of joint damaging hazard at long return periods. Damaging hazard changes by small amounts at long return periods (low frequency) therefore increases in frequency produce little change. On the other hand, changes in the strength of dependency alter the probability of co-occurrence of extreme gust and this can have a large effect on aggregate damage at long return periods.

\section{Summary}

The potentially severe and widespread damage from $\mathrm{Eu}-$ ropean windstorms stimulates interest in risk management. Companies in the re/insurance sector usually carry risks at multiple locations and hence the spatial structure, as well as the local wind intensity, is required for accurate estimates of damaging hazard caused by windstorms. Ideally, the estimates of multi-location risk would be based on windstorm events drawn from historical records. However, there are specific needs for robust estimation of the characteristics of extreme storms with return periods exceeding the observational record of such events. As a result, empirical methods for defining extreme windstorm events are rather inaccurate, and other approaches are required.

The emerging solution is based on General Circulation Models (GCMs) and nested high-resolution regional models to generate catalogues of stochastic windstorms. Though the GCMs are rooted in basic laws of dynamical motion and physical processes, the simulated near-surface peak gusts contain biases that require calibration and validation to ensure appropriate representation of extreme peril behavior. The brevity of the historical record of storms is extended by these stochastic catalogues of storms, using our understanding of atmospheric behavior integrated into the GCMs together with calibration to historical data.

The calibration and validation of the GCM-produced stochastic catalogues of storms is critical to the pricing of risk. The local wind climatology can be modeled with fairly standard statistical models such as peaks-over-threshold to produce estimates of local gusts for return periods exceeding the observation record. However, the spatial structure of the storms requires careful assessment too. We have investigated a simplified model of the spatial dependence structure of $\mathrm{Eu}-$ ropean wind storms in this analysis. Near-surface $3 \mathrm{~s}$ peak gust data were obtained from a variety of national meteorological centers and private data suppliers to form a history of European wind of the past $40 \mathrm{yr}$. Spatial maps of windstorm peak gust footprints were reconstructed for 135 of the 
most important damaging events in the past four decades. Extreme-value copulas, i.e. parametric models which couple univariate information, were fitted to the set of historical events to provide information on the spatial dependence of damaging wind storm activity in Europe.

The coupling between pairs of locations was assessed using the tail dependence coefficient. We find that within a radius of $500 \mathrm{~km}$ there is about a 1 in 2 chance that any pair of locations would simultaneously experience damaging peak gusts (defined as gusts above $20 \mathrm{~m} \mathrm{~s}^{-1}$ ), conditional on one location being hit by a damaging gust. Furthermore, the tail dependence exhibits a distinct anisotropic structure with stronger coupling in the zonal direction, which is consistent with the dominant track of extra-tropical cyclones from west to east. We present maps of observed tail dependence coefficients centered at different major cities, which may be used to assess stochastic storm catalogues.

The joint distribution of gusts was condensed into a univariate form by considering the minimum gust at a pair of locations. Two estimates of the distribution of extreme values were made: the first using the copula method, and the second by fitting the GPD directly on this scalar function. Estimates of joint information at return periods greatly exceeding data periods are inherently uncertain, and the inclusion of a different model based on directly fitting the GPD to minimum gusts can provide indications of such uncertainties. London-Paris offers an interesting case study. The dependence structure of the extreme value copula implies significantly stronger minimum gusts at return periods of 50 or more years than direct GPD fitting.

Finally, we used extreme value copulas to analyze the role of climate variability in the joint distribution of an aggregate measure of storm intensity and area across European countries. Inter-annual variability, described by the North Atlantic Oscillation, modulates the main orientation of the storm tracks and the frequency of storm events. We found that the joint distribution of UK and Danish storms is sensitive to the phase of the NAO. Years when the NAO is in its positive phase correspond to significantly higher event frequencies, which is the dominant cause of increased risk at shorter return periods. However, at return periods of $200 \mathrm{yr}$ or more, the elevated tail dependence strength between the marginal distributions of aggregated damaging hazard contributes as much to the greater risk as the increase in event frequencies.

Edited by: U. Ulbrich

Reviewed by: M. Haylock and another anonymous referee

\section{References}

Anderson, T. W. and Darling, D. A.: Asymptotic theory of certain goodness-of-fit criteria based on stochastic processes, Annals of Mathematical Statistics, 23, 193-212, 1954.

Barnes, S. L.: A techniques for maximizing details in numerical weather map analysis, J. Appl. Meteor., 3, 396-409, 1964.

Barredo, J. I.: No upward trend in normalised windstorm losses in Europe: 1970-2008, Nat. Hazards Earth Syst. Sci., 10, 97-104, doi:10.5194/nhess-10-97-2010, 2010.

Berg, D. and Bakken, H.: Copula Goodness-of-fit Test for Copulae Based on the Probability Integral Transform. Tech. Rep. 10, Department of Mathematics, University of Oslo, 2005.

Breymann, W., Dias, A., and Embrechts, P.: Dependence structures for multivariate high-frequency data in finance, Quant. Financ., 3, 1-14, 2003.

Cebrian, A. C., Denuit, M., and Lambert, P.: Analysis of bivariate tail dependence using extreme value copulas: An application to the SOA medical large claims database, Belgian Actuarial Bulletin, 3, 33-41, 2003.

Chernobay, A., Rachev, S., and Fabozzi, F.: Composites goodness of fit tests for left-truncated loss samples, Tech. rep., University of Calivornia Santa Barbara, 2005.

Coles, S.: An Introduction to Statistical Modeling of Extreme Value, Springer Series in Statistics, Springer, New York, 2001.

Cusack, S.: A 100 year time-series of windstorm losses in the Netherlands, Climatic Change, submitted, 2012.

Della-Marta, P. M., Mathis, H., Frei, C., Liniger M. A., Kleinn, J., and Appenzeller, C.: The return period of wind storms over Europe, Int. J. Climatol., 29, 437-459, doi:10.1002/joc.1794, 2008.

Falk, M., Hüsler, J., and Reiss, R.: Laws of Small Numbers: Extremes and Rare Events (2nd ed.), Birkhäuser Verlag, 2000.

Fermanian J. D.: Goodness of fit test for copulas, J. Multivariate Anal., 95, 119-152, 2005.

Friederichs, P., Göber, M., Bentizien, S., Lenz, A., and Krampitz, R.: A probabilistic analysis of wind gusts using extreme value statistics, Meteorol. Z., 18, 615-629, 2009.

Galambos J.: The Asymptotic Theory of Extreme Order Statistics, Melbourne: Krieger, 1987.

Geffroy J.: Contributions à la thèorie des valeurs extrème, Publ. Inst. Stat. Univ. Paris, 7, 37-185, 1958.

Genest, C. and Segers, J.: Rank-based inference for bivariate extreme-value copulas, Ann. Stat., 37, 2990-3022, 2009.

Genest, C., Kojadinovic, I., Neslehova J., and Yan, J.: A goodnessof-fit test for bivariate extreme-value copulas, Bernoulli, 17, 253-275, 2011.

Hutchinson, T. P. and Lai, C. D.: Continuous Bivariate Distributions, Emphasizing Applications, Adelaide: Rumsby Scientific Publishing, 1990.

Kojadinovic, I. and Yan, J.: Modeling Multivariate Distributions with Continuous Margins Using the copula R Package, J. Stat. Softw., 34, 1-20, 2007

Klawa, M. and Ulbrich, U.: A model for the estimation of storm losses and the identification of severe winter storms in Germany, Nat. Hazards Earth Syst. Sci., 3, 725-732, doi:10.5194/nhess-3725-2003, 2003.

Kushnir, Y., Robinson, W. A., Chang, P., and Robertson, A. W.: The physical basis for predicting Atlantic sector seasonal-tointerannual climate variability, J. Climate, 19, 5949-5970, 2006. 
Lamb, H.: Historic storms of the North Sea, British Isles and Northwest Europe, Cambridge Press, 1991

Ledford, A., and Tawn, A. Statistics of near independence in multivariate extreme values, Biometrika 83, 169-187, 1996.

Leadbetter, M. R. and Rootzen H.: Extremal Theory for Stochastic Processes, The Annals of Probability, 16, 431-478, 1988.

Leckebusch, G. C., Renggli, D., and Ulbrich, U.. Development and application of an objective storm severity measure for the Northeast Atlantic region, Meteorol. Z., 17, 575-587, doi:10.1127/0941-2948/2008/0323, 2008.

Mailier, P. J, Stephenson, D. B., Ferro, C. A. T., and Hodges K. I.: Serial Clustering of Extratropical Cyclones, Mon. Weather Rev., 135, 2224-2240, 2005.

Marshall, A. W.: Copulas, Marginals and Joint Distributions, in: Distributions with Fixed Marginals, and Related Topics, 213222, Hayward, CA: Institute of Mathematical Statistics, 1996.

McNeil, A. J., Frey, R., and Embrechts, P.: Quantitative Risk Management, Princeton Series in Finance, 2005.

Nelsen, R. B.: An Introduction to Copulas, Springer, 1999.

Petak, W. J. and Atkisson, A. A.: Natural hazard risk assessment and public policy: Anticipating the unexpected, Springer-Verlag, New York, 1982.

Pickands J.: Multivariate extreme value distribution. Proceedings of the 43rd Session of the International Statistics Institute, Buenos Aires, vol. 2, 859-878, The Hague: International Statistics Institute, 1981.
R Development Core Team: R: A Language and Environment for Statistical Computing. R Foundation for Statistical Computing, Vienna Austria, 2011.

Reskin, S. I.: Extreme Value, Regular Variation and Point Process, Springer, 1987.

Schölzel, C. and Friederichs, P.: Multivariate non-normally distributed random variables in climate research - introduction to the copula approach, Nonlin. Processes Geophys., 15, 761-772, doi:10.5194/npg-15-761-2008, 2008.

Sibuya, M.: Bivariate extreme statistics, Annals of the Institute of Statistical Mathematics, 11, 195-210, 1960.

Steinkohl, C., Davis, R. A., and Klüppelberg, C.: Extreme Value Analysis of Multivariate High Frequency Wind Speed Data Submitted for Publication, 2011.

Swiss Re: Natural catastrophes and man-made disasters in 2009: catastrophes claim fewer victims, insured losses fall, Swiss Re sigma report, available at: http://www.swissre.com/sigma/, 2010.

Wallace, J. M. and Gutzler, D. S.: Teleconnections in the geopotential height field during the Northern Hemisphere Winter, Mon. Weather Rev., 109, 784-812, 1981.

Wang, S. S.: Aggregation of Correlated Risk Portfolios: Models and Algorithms, CAS Committee on Theory of Risk, 1998.

Wilks, D. S.: Statistical Methods in the Atmospheric Sciences, Academic Press, 2005.

Yan, J.: Enjoy the Joy of Copulas: With a Package copula, J. Stat. Softw., 21, 1-21, 2007. 